High-precision mass measurements and production of neutron-deficient isotopes using heavy-ion beams at IGISOL

\title{
Vilen, M.
}

$2019-11-26$

Vilen , M , Kankainen , A , Baczyk , P , Canete , L , Dobaczewski , J , Eronen , T , Geldhof , S , Jokinen , A , Konieczka , M , Kostensalo , J, Moore , I D , Nesterenko, D A, Penttilä , H , Pohjalainen , I, Reponen, M , Rinta-Antila , S , de Roubin , A, Satula , W \& Suhonen , J 2019 , ' High-precision mass measurements and production of neutron-deficient isotopes using heavy-ion beams at IGISOL ' , Physical Review C , vol. 100 , no. 5 , 054333 . https://doi.org/10.1103/PhysRev

http://hdl.handle.net/10138/312071

https://doi.org/10.1103/PhysRevC.100.054333

publishedVersion

Downloaded from Helda, University of Helsinki institutional repository.

This is an electronic reprint of the original article.

This reprint may differ from the original in pagination and typographic detail.

Please cite the original version. 


\title{
High-precision mass measurements and production of neutron-deficient isotopes using heavy-ion beams at IGISOL
}

\author{
M. Vilén $\odot,{ }^{1,}{ }^{*}$ A. Kankainen $\odot,{ }^{1}$ P. Bączyk, ${ }^{2}$ L. Canete, ${ }^{1}$ J. Dobaczewski $\odot,{ }^{2,3,4}$ T. Eronen, ${ }^{1}$ S. Geldhof $\odot,{ }^{1}$ A. Jokinen, ${ }^{1}$ \\ M. Konieczka ${ }^{2}{ }^{2}$ J. Kostensalo, ${ }^{1}$ I. D. Moore ${ }^{1},{ }^{1}$ D. A. Nesterenko, ${ }^{1}$ H. Penttilä, ${ }^{1}$ I. Pohjalainen, ${ }^{1}$ M. Reponen, ${ }^{1}$ \\ S. Rinta-Antila, ${ }^{1}$ A. de Roubin, ${ }^{1}$ W. Satuła ${ }^{2},{ }^{2,4}$ and J. Suhonen ${ }^{1}$ \\ ${ }^{1}$ University of Jyväskylä, Post Office Box 35, FI-40014 Jyväskylä, Finland \\ ${ }^{2}$ Institute of Theoretical Physics, Faculty of Physics, University of Warsaw, ul. Pasteura 5, PL-02-093 Warsaw, Poland \\ ${ }^{3}$ Department of Physics, University of York, Heslington, York YO10 5DD, United Kingdom \\ ${ }^{4}$ Helsinki Institute of Physics, University of Helsinki, Post Office Box 64, FI-00014 Helsinki, Finland
}

(Received 15 July 2019; published 26 November 2019)

\begin{abstract}
An upgraded ion-guide system for the production of neutron-deficient isotopes with heavy-ion beams has been commissioned at the IGISOL facility with an ${ }^{36} \mathrm{Ar}$ beam on a ${ }^{\text {nat }} \mathrm{Ni}$ target. It was used together with the JYFLTRAP double Penning trap to measure the masses of ${ }^{82} \mathrm{Zr},{ }^{84} \mathrm{Nb},{ }^{86} \mathrm{Mo},{ }^{88} \mathrm{Tc}$, and ${ }^{89} \mathrm{Ru}$ ground states and the isomeric state ${ }^{88} \mathrm{Tc}^{m}$. Of these, ${ }^{89} \mathrm{Ru}$ and ${ }^{88} \mathrm{Tc}^{m}$ were measured for the first time. The precision of measurements of ${ }^{82} \mathrm{Zr},{ }^{84} \mathrm{Nb}$, and ${ }^{88} \mathrm{Tc}$ was significantly improved. The literature value for ${ }^{86} \mathrm{Mo}$ was verified. The measured states in ${ }^{88} \mathrm{Tc}$ were compared to shell-model calculations and additional constraints on the spins and level scheme were obtained. The masses of ${ }^{82} \mathrm{Mo}$ and ${ }^{86} \mathrm{Ru}$ have been predicted using the measured masses of their mirror partners and theoretical mirror displacement energies, resulting in more tightly bound nuclei with smaller atomic mass uncertainties than reported in the literature.
\end{abstract}

DOI: 10.1103/PhysRevC.100.054333

\section{INTRODUCTION}

Neutron-deficient nuclei in the mass region $A \approx 80-100$ provide invaluable data for understanding basic nuclear interactions. Being close to the $Z=N$ line, the protons and neutrons are filling the same orbitals, mainly the high-spin $1 g_{9 / 2}$ orbital above the subshell closure at $N=Z=40$. This opens an interesting approach to investigating proton-neutron pairing $[1,2]$ as well as isospin symmetry $[3,4]$ in nuclei. Recent theoretical calculations for mirror (MDE) or triplet displacement energies (TDE) using extended Skyrme energy density functionals with proton-neutron-mixed densities and isospin-symmetry-breaking terms have yielded good agreement with experimental data in the lower mass region $[3,4]$. For example, with next-to-leading-order isospin-symmetrybreaking terms included, the root mean square deviation (RMSD) to experimental data for $T=1$ MDEs is $180 \mathrm{keV}$ and only around $65 \mathrm{keV}$ for TDEs [4]. Above $A \approx 70$, the available experimental data for MDEs or TDEs is rather limited. There, these new calculations can provide predictions for the more exotic mirror partners in the isobaric doublets or triplets.

The neutron-deficient region close to $A \approx 80$ is known for shape coexistence and deformation. For example, the $N=$ $Z=40$ nucleus ${ }^{80} \mathrm{Zr}$ is one of the most deformed nuclei known so far with a quadrupole deformation of $\beta_{2} \approx 0.4$ [5]. Total-Routhian-surface calculations have indicated that the

*markus.k.vilen@student.jyu.fi $g_{9 / 2}$ shell plays an important role in the shape evolution, with spherical, prolate, oblate, and triaxial shapes predicted [6]. Even possible tetrahedral deformation has been proposed to exist in the region [7]. The onset of deformation is reflected in nuclear binding energies [8], and therefore new precision mass measurements can shed light on the shape changes in the $A=80-100$ region.

The masses of neutron-deficient nuclei are also relevant for the astrophysical rapid proton $(r p)$ capture process occurring in type I x-ray bursts $[9,10]$. For reliable calculations of the produced light curves and burst ashes, the masses should be known with a precision of around $10 \mathrm{keV}$ [9]. Previous mass measurements at JYFLTRAP [11-13] and SHIPTRAP [12,14] have revealed large deviations up to $1 \mathrm{MeV}$ to the earlier literature values. Before the Penning trap era, the masses were mainly based on $\beta$-decay endpoint energies which are prone to underestimations of the $Q$ values and hence the mass values.

Neutron-deficient nuclei in the $A=80-100$ region are rich in long-living isomeric states. Nucleons in the high-spin $1 g_{9 / 2}$ orbital can pair up from low to high spins, and odd nucleons can also occupy the low-spin $2 p_{1 / 2}$ orbital below, creating plenty of opportunities for spin-trap isomers. If a measured state is wrongly assigned as the ground state when in reality it is the isomer or a mixture of states, it can cause a substantial offset and lead to biased $r p$-process calculations. The excitation energies for low-lying, $\beta$-decaying isomeric states have been very difficult to measure due to the lack of resolving power in available mass-measurement techniques. Recently, the phase-imaging ion cyclotron resonance (PI-ICR) technique $[15,16]$ has made it possible to measure isomers 
with very low excitation energies $\left(E_{x} \geqslant 10 \mathrm{keV}\right)$, providing valuable data for understanding nucleon-nucleon interactions and single-particle properties in nuclei.

Many neutron-deficient nuclei in the $A=80-100$ region have been challenging to produce at conventional ISOL facilities due to their refractory nature. One solution to the problem is the fast (sub-ms) and chemically insensitive method developed at the Ion Guide Isotope Separator On-Line (IGISOL) facility for the production of low-energy radioactive ion beams for nuclear physics experiments [17-19]. Although this ion-guide technique has proven to be a valuable tool in producing a great variety of rare isotope beams covering a large portion of the nuclear chart, heavy neutron-deficient nuclei lying further away from stability have been more difficult to access. The most commonly used ion-guide types at IGISOL have been optimized for either light-ion-induced fusion or proton-induced fission reactions [18]. There, the target foil is mounted in direct contact with the buffer gas and the primary beam traverses through the gas cell. For heavy-ion beams this is not possible because the primary beam would create a substantial number of buffer-gas ion-electron pairs which are detrimental to the overall efficiency. To tackle this problem, a heavy-ion ion-guide isotope separator online (HIGISOL) method [19-21] employing a shadow gas-cell technique [22] was developed at IGISOL. In this method, the target is located in front of the ion guide and the primary beam is stopped before entering the gas cell. The reaction products are emitted at sufficiently large angles to bypass the beam dump and enter the gas cell through a thin window. Recently, the HIGISOL ion guide system was upgraded and used in an online experiment for the first time. In this article, we report on this commissioning experiment which was used to produce neutron-deficient refractory isotopes for precision mass measurements, improving the knowledge of the mass surface in the mass region $A \approx 80-90$.

\section{EXPERIMENTAL METHODS}

\section{A. Production using the upgraded HIGISOL}

The heavy neutron-deficient nuclei were produced employing a $260-\mathrm{pnA}, 222-\mathrm{MeV}{ }^{36} \mathrm{Ar}^{8+}$ beam impinging on a ${ }^{n a t} \mathrm{Ni}$ target with a thickness of $4 \mu \mathrm{m}$ at the IGISOL facility [18,24]. The upgraded HIGISOL system (see Fig. 1) houses two degrader foils that can be used to fine-tune the primary beam energy. However, in this work the full beam energy was used. The target was located outside the gas cell next to the degrader foils. The primary beam was stopped right after the target using a small cylindrical graphite beam dump mounted in front of the gas cell. This shielded the buffer gas from excessive ionization. In the new HIGISOL system, the target is mounted on a rotating wheel and the degrader foils are put on frames that can be moved back and forth in order to allow the thermal power of the primary beam to be dissipated over a larger surface area. The electronics for the platform were upgraded and a new control system was constructed utilizing a combination of a Raspberry Pi computer and an Arduino microcontroller. The rotational frequency of the target wheel as well as the position of the target wheel and degrader foils

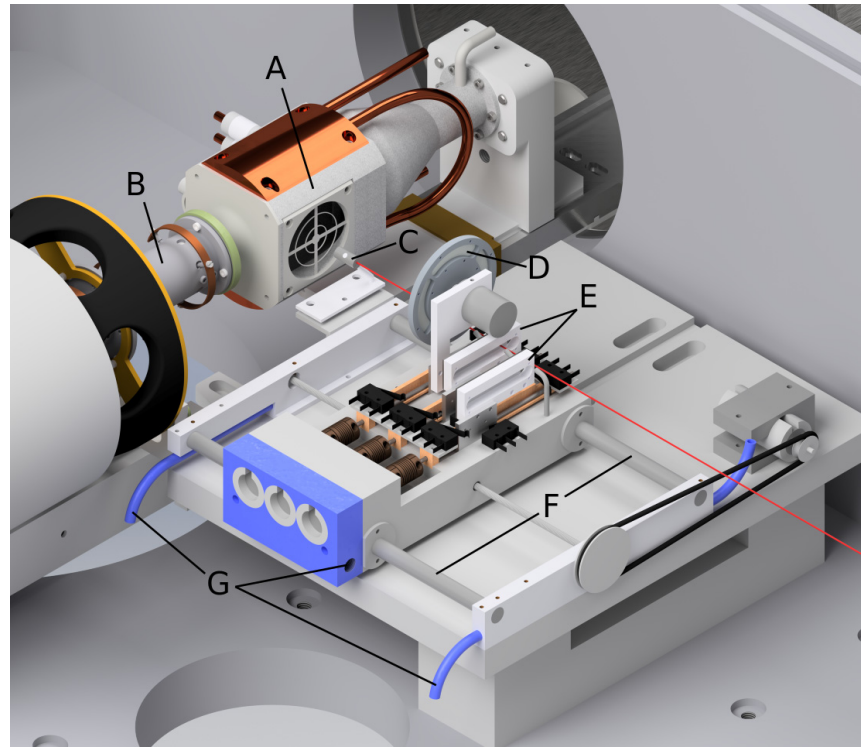

FIG. 1. The new HIGISOL system: (a) ion-guide gas cell, (b) SPIG [23], (c) beam dump, (d) target wheel, (e) degrader holder, (f) rails for distance adjustment, and (g) coolant line.

can now be remotely controlled using JAVA-based software. Additionally, the whole platform can be remotely moved closer or further away from the gas cell, in parallel to the primary beam axis, based on the position information provided by the system. This production method has been utilized at IGISOL in the past using two mechanical designs. For further details, the reader is referred to Ref. [20] for the original design and to Ref. [21] for the second iteration of the system design.

The choice of primary beam energy was made based on fusion-evaporation cross-section simulations using PACE4 [25], HIVAP [26], and NRV [27] codes. An example of an ion yield contour for an ${ }^{36} \mathrm{Ar}^{8+}$ beam on a ${ }^{\text {nat }} \mathrm{Ni}$ target obtained with the PACE4 [25] code is presented in Fig. 2. The position of the yield maximum changes as a function of primary beam energy in $\left(E_{r}, \theta\right)$ space, where $E_{r}$ is the energy of recoiling reaction products and $\theta$ is the deflection angle. Reaction products have their yield maxima at slightly different, nonzero angles. Therefore, the distance between the gas cell and the target wheel needs to be optimized for each ion of interest so that the recoiling reaction products can enter the ion-guide gas cell through its entrance window and are not implanted into the beam dump. In addition to the angle of the products, the kinetic energy of the reaction products has to be taken into account. Ions of interest must have enough energy after the target to go through the $2.17-\mu \mathrm{m}$-thick Havar window and then be stopped and thermalized within the buffer gas (helium) volume of the HIGISOL ion guide. Therefore, the pressure (stopping power) of the buffer gas needs to be adjusted so that the range of the ions does not exceed the inner diameter of the HIGISOL gas cell (approximately $6 \mathrm{~cm}$ ). In order to accommodate different recoil energies and angular distributions resulting from the choice of primary beam energy and target, both the distance between the target and the gas cell, as well as buffer gas pressure, can be adjusted 


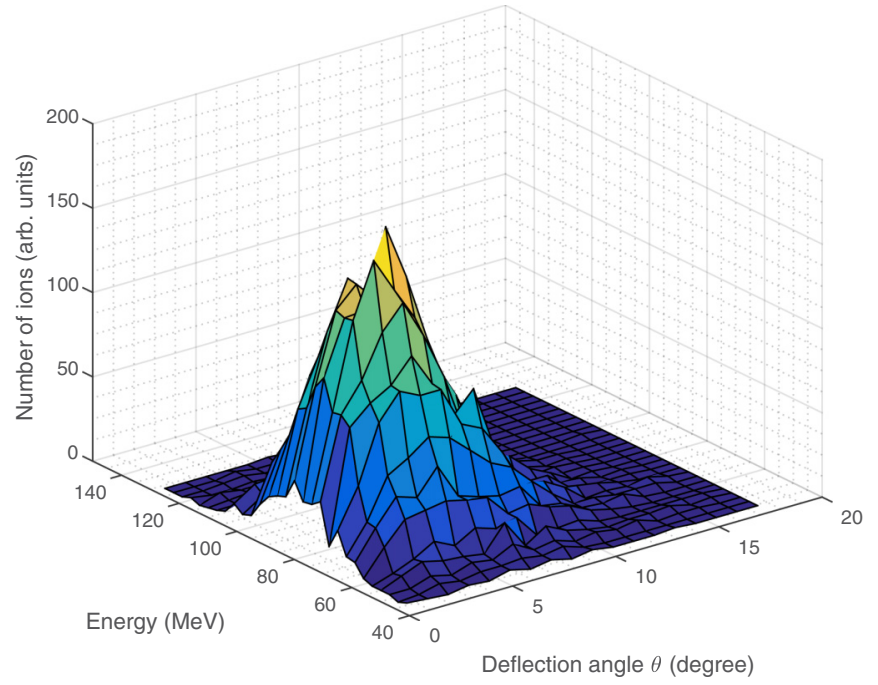

FIG. 2. Yield distribution (in arbitrary units) as a function of recoiling energy $E_{r}$ and angle $\theta$ for a $228.60-\mathrm{MeV}^{36} \mathrm{Ar}$ beam on a ${ }^{\text {nat }} \mathrm{Ni}$ target summed over all reaction products using the PACE4 program.

in the new system. These degrees of freedom are sufficient for optimizing the system for different primary beam energies for a variety of reaction channels. In this experiment, the helium pressure was typically around 240 mbar.

Reaction products stopped and evacuated from the ion guide gas cell were collected and guided toward the highvacuum region of the mass separator using a radio-frequency sextupole ion guide (SPIG) [23], with a typical charge state being $q=+e$. The ions were accelerated to $30 \mathrm{keV}$ and mass separated using a dipole magnet before stopping, cooling, and bunching in the radio-frequency quadrupole cooler-buncher (RFQ) [28]. The ion bunches from the RFQ were injected into the double-Penning-trap mass spectrometer, JYFLTRAP [29].

\section{B. Mass measurement techniques}

Masses of the nuclides of interest were measured utilizing time-of-flight ion-cyclotron resonance (TOF-ICR) [30,31] and phase-imaging ion-cyclotron resonance (PI-ICR) [15] techniques. Measured ions were prepared in the purification trap via the mass-selective buffer gas cooling method [32], which is capable of providing isobarically purified ion samples. Masses were determined in the precision trap by measuring the ion's cyclotron frequency $v_{c}=q B /(2 \pi m)$, where $q$ and $m$ are the charge and mass of the ion, respectively, and $B$ is the magnetic field strength. The magnetic field strength was acquired by interleaving measurements of ions with well-known masses $\left(v_{c, \text { ref }}\right)$ before and after each measurement of the ion of interest $\left(v_{c}\right)$ and interpolating the field strength at the time of the ion of interest measurement. Identical excitation patterns were applied in the precision trap for the ion of interest and the reference ion in order to minimize the magnitude of any systematic errors due to different ion species. In this work, ${ }^{85,87} \mathrm{Rb}^{+}$ions were used as references.

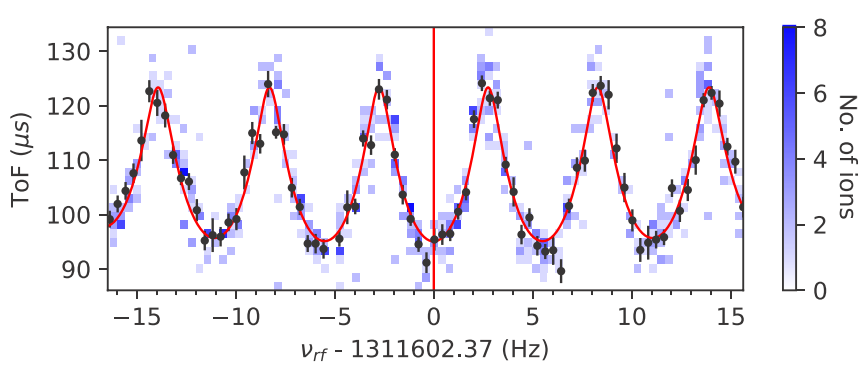

FIG. 3. A typical time-of-flight resonance of ${ }^{82} \mathrm{Zr}^{+}$using Ramsey excitation pattern $25-150-25 \mathrm{~ms}$ (on-off-on). The black points with error bars represent the mean time of flight for each scanned quadrupolar excitation frequency $v_{r f}$. Background shading indicates the total number of ions in each time-of-flight bin. The red solid line is a fit of the theoretical curve [33] on the data points.

Two excitation schemes were utilized in the TOF-ICR measurements. A 200-ms quadrupole excitation pulse was used for ${ }^{86} \mathrm{Mo}$ and ${ }^{88} \mathrm{Tc}$ whereas the remaining masses were determined using the method of time-separated oscillatory fields $[33,34]$ with $25-750-25 \mathrm{~ms}$ (on-off-on) excitation pattern for ${ }^{84} \mathrm{Nb}$ and $25-150-25 \mathrm{~ms}$ (on-off-on) for ${ }^{82} \mathrm{Zr}$ and ${ }^{89} \mathrm{Ru}$. For these cases, the position of the center fringe was first verified via a measurement with a single conversion pulse. An example of a time-of-flight resonance of ${ }^{82} \mathrm{Zr}$ is presented in Fig. 3.

${ }^{88} \mathrm{Tc}$ was first measured using the TOF-ICR technique in order to improve the precision of the literature value of its ground-state mass. This was followed by a PI-ICR measurement in which the energy separation between the ground state and presumed first isomeric state was measured. The mass difference between the two states is too low to result in two separate peaks in the TOF-ICR measurement with 200-ms quadrupolar excitation.

In the PI-ICR measurements, ion samples were prepared in the purification trap in the same way as with the TOF-ICR measurements. Ions were injected into the precision trap and the amplitude of residual coherent magnetron and axial motion was damped using RF fields of suitable frequency, phase, and amplitude in preparation for the actual measurement. As ions are injected into the precision trap, they acquire a nonzero amplitude for the magnetron and axial eigenmotions. If not addressed, the nonzero amplitudes would adversely affect the measurement precision. Therefore, for each eigenmotion, a dipole pulse with a suitable amplitude $\pi$ rad out of phase with the motion is applied in order to reduce the motion amplitude.

After the residual eigenmotion amplitudes were damped, a dipole excitation pulse at the modified cyclotron frequency $v_{+}$was applied followed by a phase accumulation time of $200 \mathrm{~ms}$ and a conversion pulse to convert the ion motion into magnetron motion. Finally, the ions were extracted out of the trap toward a position-sensitive delay-line MCP detector. This measurement cycle was followed by one with slightly modified timings. The second cycle was identical to the first one with the exception of the phase accumulation time being after the conversion pulse rather than before. Additionally, the center point of the precision trap was projected onto the detector. These three measurement cycles allowed us to 
calculate the mass difference between the ground and isomeric states of ${ }^{88} \mathrm{Tc}$. For a more detailed discussion on this measurement technique, the reader is referred to Refs. [15,16], where the excitation scheme used in this work is presented as Scheme 2.

\section{Data analysis}

In the case of the TOF-ICR method, the cyclotron frequency $v_{c}$ was measured multiple times for each nuclide. Each cyclotron frequency measurement was corrected, whenever possible, for shifts due to having multiple ions in the precision trap at the same time, as described in Ref. [35]. Data available for ions of interest were not sufficient to allow for this kind of corrections to be made. However, all reference ion species were sufficiently abundant for applying the correction. Additionally, a correction due to $B$-field fluctuations, $\delta_{B}\left(v_{c, \text { ref }}\right) / v_{c, \text { ref }}=\left(8.18 \times 10^{-12} / \mathrm{min}\right) \Delta t$ [36], was applied to each interpolated reference ion frequency, where $\Delta t$ is the time between consecutive reference measurements, followed by calculation of frequency ratio $(r)$ for each measurement. Atomic masses were calculated according to

$$
\begin{gathered}
r=\frac{v_{c, \text { ref }}}{v_{c}}=\frac{m_{\text {ion of interest }}}{m_{\text {reference ion }}} \\
m=\frac{v_{c, \text { ref }}}{v_{c}}\left(m_{\text {ref }}-m_{e}\right)+m_{e},
\end{gathered}
$$

where the cyclotron frequencies $v_{c}$ are for the studied singly charged ions and the masses are expressed as atomic masses unless stated otherwise in subscripts. Electron binding energies were neglected as such corrections are beyond the precision of our measurements.

A weighted mean of frequency ratios along with internal and external errors [37] were calculated, and the larger of the two was chosen as the uncertainty of the mean. In most cases, the Birge ratios were smaller than 1, indicating that statistical uncertainties of individual measurements were conservative. Finally, a mass-dependent uncertainty $\delta_{m}(r) / r=[2.2(6) \times$ $\left.10^{-10} / u\right] \Delta m$ [36] was added to the frequency ratio error, where $\Delta m$ is the mass difference of the ion of interest and the reference ion. The applied data analysis process takes into account all systematic uncertainties that had been quantified at the time of the measurements. A detailed discussion on the different sources of systematic uncertainties at JYFLTRAP is to follow this article [38].

Data measured using the PI-ICR technique were used to determine the energy difference between the ground state and the presumed first isomeric state of ${ }^{88} \mathrm{Tc}$ (see Fig. 4). The angle between the accumulated phases of magnetron and reduced cyclotron radial motions was calculated from multiple measurements for both the ground and isomeric states, followed by the calculation of the cyclotron frequency using

$$
v_{c}=\frac{\phi+2 \pi\left(n_{+}+n_{-}\right)}{2 \pi t},
$$

where $\phi$ is the angle between the positions of the accumulated magnetron and cyclotron phase spots projected on the detector and $n_{+}$and $n_{-}$are the number of full revolutions ions completed in corresponding timing patterns within the phase accumulation time $t$. A frequency ratio between the isomeric

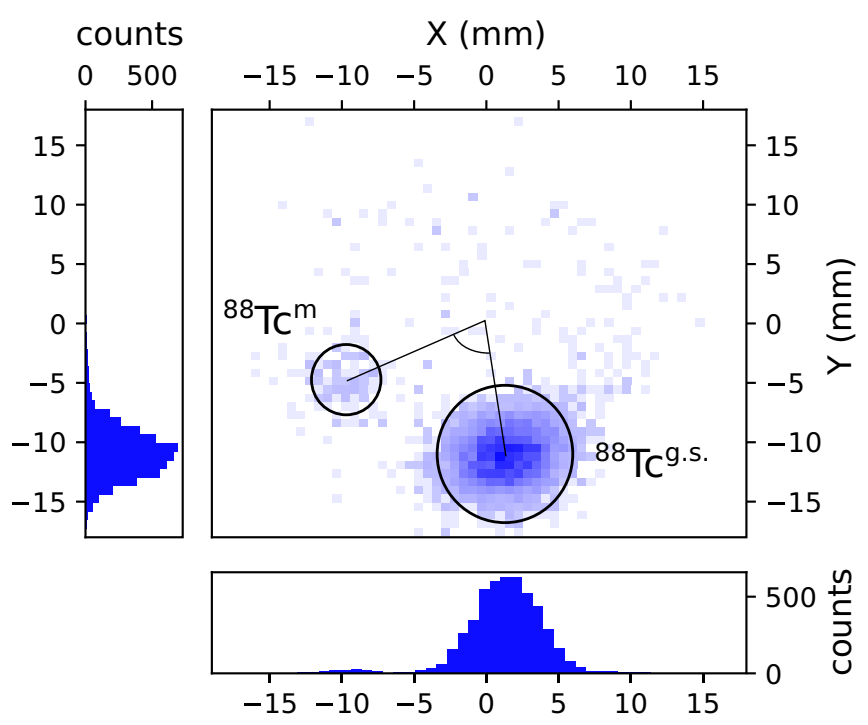

FIG. 4. Projection of reduced cyclotron motion of ${ }^{88} \mathrm{Tc}^{+}$ions on the position-sensitive detector using the PI-ICR technique. The ground state and isomer are clearly separated using 200-ms phase accumulation time.

and ground states, $r=v_{c, g s} / v_{c}$, is, was calculated for each measurement. A weighted mean of the frequency ratios was used to determine the mass difference between the two states, i.e., the excitation energy of the isomer $E_{x}$, using

$$
E_{x}=\left(m_{i s}-m_{g s}\right) c^{2}=(r-1)\left(m_{g s}-m_{e}\right) c^{2},
$$

where $m_{g s}$ is the mass of the ground state determined in this work using the TOF-ICR technique. Inner and outer errors were determined, and the larger one was chosen as the error of the mean. Systematic uncertainties of the PI-ICR measurements with JYFLTRAP were studied in Ref. [16]. Systematic uncertainties discussed in Ref. [16] were found to be insignificant compared to the statistical uncertainty of this work.

One additional systematic uncertainty that was accounted for in this work is the shift of measured frequency as a function of the angle between the accumulated phase spots in the PI-ICR method due to distortions of ion-motion projection onto the detector. As shown in Fig. 4, there was a nonzero angle between the isomeric and ground states. For the purpose of characterizing the effect of the angle, another measurement was performed with stable ${ }^{87} \mathrm{Rb}$ ions where the $v_{c}$ frequency was determined using ${ }^{87} \mathrm{Rb}$ as a reference. The positions of the phase spots on the detector were tuned to match the ${ }^{88} \mathrm{Tc}$ measurement. In an ideal case, a measurement like this would result in a frequency ratio $r=1$. However, the measurement resulted in

$$
r-1=-3.7(2) \times 10^{-8} .
$$

This systematic shift was corrected for in the results and, additionally, added quadratically as a systematic uncertainty. In the case of the PI-ICR measurement between ${ }^{88} \mathrm{Tc}$ and ${ }^{88} \mathrm{Tc}^{m}$, the effect of having multiple ion species in the precision trap could not be accounted for due to the low production rate of the isomeric state. 
TABLE I. Frequency ratios $(r)$ and mass-excess values $\left(M E_{\text {JYFL }}\right)$ determined in this work with JYFLTRAP and compared with AME16 [39]. All measurements were done with singly-charged ions. The reference masses, ${ }^{85} \mathrm{Rb}(M E=-82167.331(5)[39])$ and ${ }^{87} \mathrm{Rb}(M E=$ -84597.791(6) [39]) were adopted from AME16, and \# signs indicate extrapolated values therein. The differences between the this work and AME16 are listed in the last column. The excitation energy for ${ }^{88} \mathrm{Tc}^{m}, E_{x}=70.4(31) \mathrm{keV}$, was determined for the first time; see text for details.

\begin{tabular}{lccccc}
\hline \hline Nuclide & Reference & $r=v_{c, \text { ref }} / v_{c}$ & $M E_{\text {JYFL }}(\mathrm{keV})$ & $M E_{\mathrm{AME} 16}(\mathrm{keV})$ & $\Delta M E_{\text {JYFL}-\mathrm{AME} 16}(\mathrm{keV})$ \\
\hline${ }^{82} \mathrm{Zr}$ & ${ }^{85} \mathrm{Rb}$ & $0.96490356(2)$ & $-63613(2)$ & $-63631(12)$ & $17(12)$ \\
${ }^{84} \mathrm{Nb}$ & ${ }^{85} \mathrm{Rb}$ & $0.988488167(5)$ & $-61193.8(4)$ & $-61219(13)$ & $25(13)$ \\
${ }^{86} \mathrm{Mo}$ & ${ }^{85} \mathrm{Rb}$ & $1.01200528(6)$ & $-64112(5)$ & $-64113(5)$ & $-2(6)$ \\
${ }^{88} \mathrm{Tc}$ & ${ }^{87} \mathrm{Rb}$ & $1.01178955(5)$ & $-61670(4)$ & $-61681(149)$ & $11(149)$ \\
${ }^{88} \mathrm{Tc}^{m}$ & ${ }^{88} \mathrm{Tc}$ & $1.00000086(4)^{\mathrm{a}}$ & $-61600(5)$ & $-61680(340) \#[40]$ & $-80(340) \#$ \\
${ }^{89} \mathrm{Ru}$ & ${ }^{85} \mathrm{Rb}$ & $1.0474089(3)$ & $-58372(21)$ & $-58260(298) \#$ & $-112(299) \#$ \\
\hline \hline
\end{tabular}

${ }^{\text {a }}$ Measured using the PI-ICR technique.

\section{RESULTS AND DISCUSSION}

\section{A. Results and comparison to literature}

In this work, a total of six masses were measured: ${ }^{82} \mathrm{Zr}$, ${ }^{84} \mathrm{Nb},{ }^{86} \mathrm{Mo},{ }^{88} \mathrm{Tc},{ }^{88} \mathrm{Tc}^{m}$, and ${ }^{89} \mathrm{Ru}$. Of these, ${ }^{88} \mathrm{Tc}^{m}$ and ${ }^{89} \mathrm{Ru}$ were measured for the first time. The mass-excess value for ${ }^{89} \mathrm{Ru}$ is 14 times more precise and $112(299) \mathrm{keV}$ lower than the AME16 extrapolation. A more detailed study of the mass surface and separation energies is presented in Sec. IIIC. The excitation energy of the isomeric state in ${ }^{88} \mathrm{Tc}^{m}$ was also determined for the first time and it is discussed in Sec. III B. Precisions for the mass values of ${ }^{82} \mathrm{Zr},{ }^{84} \mathrm{Nb}$, and ${ }^{88} \mathrm{Tc}$ were significantly improved and the mass of ${ }^{86} \mathrm{Mo}$ was verified in this work. The resulting mass-excess values along with frequency ratios and literature values for the reference ions are presented in Table I.

The mass values determined for ${ }^{86} \mathrm{Mo}$ and ${ }^{88} \mathrm{Tc}$ in this work agree well with the previous literature values from Penning-trap measurements. The mass-excess value of ${ }^{86} \mathrm{Mo}$, $-64112(5) \mathrm{keV}$, agrees almost perfectly with the SHIPTRAP Penning trap measurement $-64110(4) \mathrm{keV}$ [14]. Also the mass excess value determined in this work for ${ }^{88} \mathrm{Tc}$, $-61670(4) \mathrm{keV}$, is in a good agreement with AME16. The AME16 value, -61681(149) keV [39], is mainly based on an earlier measurement at JYFLTRAP [12] where the authors measured an unidentified state at $-61679.1(3.8) \mathrm{keV}$. The isomeric state could not be separated with the TOF-ICR technique used at that time, which led the authors to assign the measured state as the high-spin ground state with an increased uncertainty of $87 \mathrm{keV}$ based on analogy with the neighboring odd-odd nuclide ${ }^{90} \mathrm{Tc}$.

Of the studied nuclides, ${ }^{82} \mathrm{Zr}$ and ${ }^{84} \mathrm{Nb}$ have both been measured at the CSRe storage ring [41] using isochronous mass spectrometry and were already included in AME16 [39] as private communications. Our more precise measurements suggest that ${ }^{82} \mathrm{Zr}$ is $17(12) \mathrm{keV}$ and ${ }^{84} \mathrm{Nb} 25(13) \mathrm{keV}$ less bound than in AME16, resulting in a disagreement of more than $1 \sigma$ in both cases. Since the AME16 values were based on the CSRe storage ring measurements [41], we decided to do a more thorough comparison between the Penning-trap and storage-ring measurements to find out if there is a systematic deviation between the two methods.

First, we gathered all published CSRe results and checked which nuclides had been measured also with a Penning trap.
All in all, there were 17 nuclides available for the comparison with the CSRe publications [41-45]. These include publications on ${ }^{37} \mathrm{~K}$ [46] and ${ }^{39} \mathrm{Ca}$ [47] from ISOLTRAP, ${ }^{42} \mathrm{Ti}$ [48], ${ }^{45} \mathrm{~V}$ [49], ${ }^{49} \mathrm{Mn}$ [49], ${ }^{52} \mathrm{Co}$ [50], ${ }^{52} \mathrm{Co}^{m}$ [50], ${ }^{53} \mathrm{Co}$ [51], ${ }^{55} \mathrm{Ni}[51],{ }^{57} \mathrm{Cu}[51],{ }^{59} \mathrm{Zn} \mathrm{[51]},{ }^{82} \mathrm{Zr}$ (this work), ${ }^{84} \mathrm{Nb}$ (this work) from JYFLTRAP, ${ }^{56} \mathrm{Cu}$ [52] from LEBIT, and ${ }^{90} \mathrm{Ru}$ [12,53] from CPT, SHIPTRAP, and JYFLTRAP. As can be seen from Fig. 5, Penning-trap measurements seem to give on the average around $20 \mathrm{keV}$ higher mass values than CSRe. It is also noteworthy that only 4 out of 17 measurements give lower mass values than CSRe.

The obtained $\chi_{n}$ value between the two methods, calculated according to

$$
\chi_{n}=\sqrt{\frac{1}{N} \sum_{i=1}^{N} \frac{\left(M E_{\mathrm{trap}, i}-M E_{\mathrm{CSRe}, i}\right)^{2}}{\delta M E_{\mathrm{trap}, i}^{2}+\delta M E_{\mathrm{CSRe}, i}^{2}},}
$$

is $\chi_{n}=1.54$ without the revised CSRe values for ${ }^{45} \mathrm{~V}$ and ${ }^{49} \mathrm{Mn}$ [45], and $\chi_{n}=1.47$ using the revised values. In both

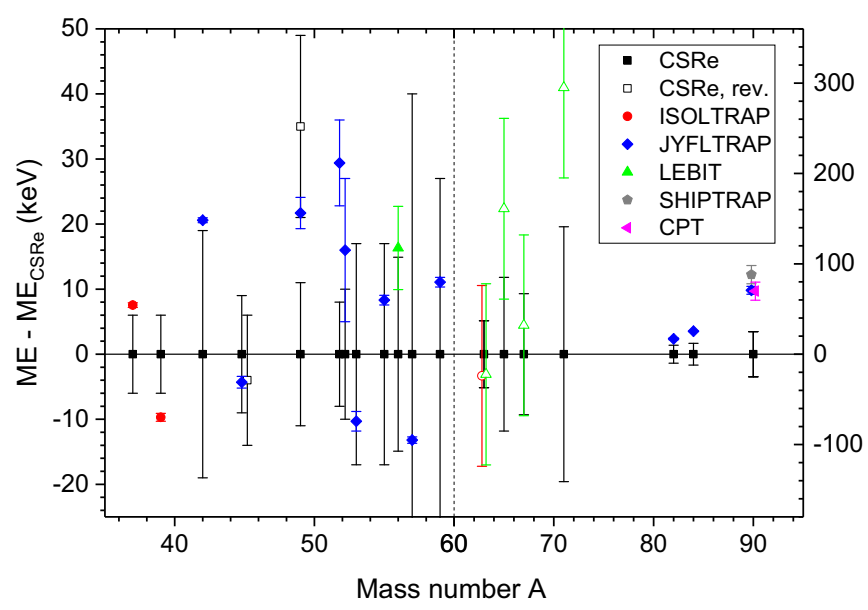

FIG. 5. Comparison of Penning-trap measurements performed at ISOLTRAP, JYFLTRAP, LEBIT, SHIPTRAP, and CPT to the results from CSRe. The open circles and triangles denote the masses estimated based on the masses of ${ }^{63} \mathrm{Ga},{ }^{65} \mathrm{Ge},{ }^{67} \mathrm{As}$, and ${ }^{71} \mathrm{Br}$ and mirror displacement energies. The revised CSRe values for ${ }^{45} \mathrm{~V}$ and ${ }^{49} \mathrm{Mn}$ are shown by open squares. Note the different $y$-axis scale after $A=60$ (shown on the right-hand side). 
cases, the $\chi_{n}$ value is above the limit $\chi_{n}=1+1 / \sqrt{2 N}=$ 1.18. Therefore, the deviation between the trap and CSRe measurements cannot be explained solely by statistical fluctuations of the two datasets. Note that here only the JYFLTRAP measurement for ${ }^{90} \mathrm{Ru}$ was taken into account in order to not triple count the CSRe measurement.

In addition to the above mentioned measurements, estimations for ${ }^{63} \mathrm{Ge},{ }^{65} \mathrm{As},{ }^{67} \mathrm{Se}$, and ${ }^{71} \mathrm{Kr}$ can be obtained from the mirror displacement energies (MDE) and precise Penningtrap measurements of ${ }^{63} \mathrm{Ga}$ [54] at ISOLTRAP as well as ${ }^{63} \mathrm{Ga}$ [55], ${ }^{65} \mathrm{Ge}$ [55], ${ }^{67} \mathrm{As}$ [55], and ${ }^{71} \mathrm{Br}$ [56] at LEBIT. These are shown as open circles in Fig. 5. Above $A=63$, all the Penning-trap values are clearly higher than what the CSRe measurements yield.

\section{B. Isomeric state in ${ }^{88} \mathrm{Tc}$}

In this work, we determined the excitation energy and the mass-excess value for the isomeric state in ${ }^{88} \mathrm{Tc}$ for the first time using the recently implemented PI-ICR technique at JYFLTRAP. Moreover, this enabled us to remove the uncertainty regarding the isomeric contamination and therefore considerably improve the precision of the ground-state mass. The mass excess of ${ }^{88} \mathrm{Tc}^{m}$ was determined using a combination of two measurements. First, the dominantly produced ground state was measured using the TOF-ICR technique. Second, the less-populated isomeric state was successfully separated from the dominant state using the PI-ICR technique. The latter was used to measure the excitation energy of the isomeric state and to determine the relative abundance of the two states. It was found that the ground state accounted for $97(1) \%$ of ions detected after the Penning traps. The excitation energy of the isomeric state was measured to be 70.4(31) keV.

Based on the yield ratio and the excitation energy of the isomeric state, i.e., frequency ratio of the two states, a correction was made for the TOF-ICR measurement of ${ }^{88} \mathrm{Tc}$ to account for the presence of the weakly populated isomeric state. The applied correction was calculated using

$$
v_{c, \text { measured }}=a v_{c, g s}+(1-a) v_{c, i s},
$$

leading to

$$
\frac{v_{c, \text { measured }}}{v_{c, g s}}=a+(1-a) \frac{v_{c, i s}}{v_{c, g s}},
$$

where $a$ is the fraction of ions in the ground state. Using frequency ratio $v_{c, i s} / v_{c, g s}$ from the PI-ICR measurement, we get

$$
v_{c, \text { measured }}=\left(1-25.7 \times 10^{-9}\right) v_{c, g s} .
$$

Applying this correction to all TOF-ICR measurements of ${ }^{88} \mathrm{Tc}$ resulted in a shift of $-2.1 \mathrm{keV}$ to the ground-state mass excess. In order to be conservative with our results, the error of the ground-state mass excess was increased by the same amount. The mass excess and frequency ratio values of ${ }^{88} \mathrm{Tc}$ and ${ }^{88} \mathrm{Tc}^{m}$ presented in Table I have this correction included.

In addition to the states measured in this work, there is a second isomeric state with a very short half-life, $T_{1 / 2}=$ $146 \mathrm{~ns}$, listed in the latest NUBASE16 evaluation of nuclear properties [40]. The half-life of the state is several orders of magnitude below what is reachable via Penning-trap mass measurements. Therefore, we conclude that our results correspond to states listed as the ground state and the longerlived isomeric state in Ref. [40], with half-lives 6.4 and $5.8 \mathrm{~s}$, respectively.

The order of the three lowest states in ${ }^{88} \mathrm{Tc}$ has remained unclear. The presumed ground state of ${ }^{88} \mathrm{Tc}$ was observed for the first time in 1991, and assigned as $7,8^{+}$due to observed feeding of $8^{+}$and $7^{-}$states in ${ }^{88} \mathrm{Mo}$ [60]. The $\beta$-decay study of Odahara et al. [61] suggested spins of $3^{+}$and $6^{+}$ for the detected states with half-lives of 5.8(2) and 6.4(8) s, respectively. The isomeric states of ${ }^{88} \mathrm{Tc}$ were further studied by Garnsworthy et al. [62]. Based on the observed 95-keV transition, which they assign tentatively as $4^{+} \rightarrow 2^{+}$, and by comparing with the shell-model calculations using the GrossFrenkel interaction [63] in a $1 g_{9 / 2}-2 p_{1 / 2}$ model space, they suggest that the $\left(5^{+}, 6^{+}, 7^{+}\right)$state listed as the ground state in Ref. [40] would in reality be the first isomeric state with spin $6^{+}$. The ground state would then be $2^{+}$fed by the observed 95-keV transition.

The excitation energy measured in this work, $E_{x}=$ $70.4(31) \mathrm{keV}$, is lower than the observed $95-\mathrm{keV}$ transition energy for the $4^{+} \rightarrow 2^{+}$(or $2^{+} \rightarrow 4^{+}$) transition in Ref. [62]. Therefore, either the $4^{+}$or $2^{+}$state should lie above the isomeric state observed in this work. We studied possible options for the order of the states: (i) $6^{+}\left(E_{x}=0 \mathrm{keV}\right), 2^{+}$ $\left(E_{x}=70 \mathrm{keV}\right), 4^{+}\left(E_{x}=165 \mathrm{keV}\right)$; (ii) $6^{+}\left(E_{x}=0 \mathrm{keV}\right), 4^{+}$ $\left(E_{x}=70 \mathrm{keV}\right), 2^{+}\left(E_{x}=165 \mathrm{keV}\right)$; (iii) $2^{+}\left(E_{x}=0 \mathrm{keV}\right), 6^{+}$ $\left(E_{x}=70 \mathrm{keV}\right), 4^{+}\left(E_{x}=95 \mathrm{keV}\right)$; or (iv) $4^{+}\left(E_{x}=0 \mathrm{keV}\right)$, $6^{+}\left(E_{x}=70 \mathrm{keV}\right), 2^{+}\left(E_{x}=95 \mathrm{keV}\right)$. We calculated halflives for the isomeric states using Weisskopf estimates. Option (i) is not plausible since the $165-\mathrm{keV} E 2$ transition from $4^{+}$ to $6^{+}$should be faster and more intense than the $95-\mathrm{keV}$ transition observed clearly in Ref. [62], and therefore should have been detected in the earlier works $[60,62]$. The fact that no other $\gamma$ rays than the $95-\mathrm{keV}$ transition was observed from the 146(12)-ns isomeric state in Ref. [62] is revealing: The energy difference between the states has to be so small that it was below the detection threshold in Ref. [62].

For option (ii), the 70-keV E2 transition would be too fast for the state to be detected with a Penning trap. The same is true for option (iv), and it can be excluded. Therefore, the most likely option is (iii), with $2^{+}$as the ground state and $6^{+}$as the isomeric state just below the $4^{+}$state (see Fig. 6). This result is somewhat surprising because $97(1) \%$ of the ions detected after the Penning traps belonged to the ground state of ${ }^{88} \mathrm{Tc}$. Heavy-ion fusion-evaporation reactions tend to predominantly populate higher spin states. As such, the production ratio of the states would favor $6^{+}$as the ground state, i.e., option (i). Note that the half-lives of the ground and isomeric states are roughly similar [61] and long compared to the trap cycles, and cannot therefore explain the dominance of the ground-state ions.

Shell-model calculations were performed in order to compare our experimental results with theoretical models. Three nuclear interactions were employed, slgt0 [57], jun45 [58], and jj44b [59], using the same model space as in Ref. [62], $1 p_{1 / 2}-0 g_{9 / 2}$. Acquired level schemes, presented in Fig. 6, do 
(a)

(b) (c)

(d) (e)

$$
\begin{aligned}
& 222=4^{265-4^{+}} \text {2 }^{+} \\
& 170-4^{+} \\
& \begin{array}{c}
95 \longrightarrow{ }^{+} \\
\mathrm{X}=70.4 \longrightarrow 6^{+} \\
\end{array}
\end{aligned}
$$

FIG. 6. Experimental (a) and theoretical excitation energies [(b)-(f)] for ${ }^{88} \mathrm{Tc}$. Based on this work, the ground state is most likely $2^{+}$and the first isomeric state $6^{+}$. The theoretical calculations employed the $1 p_{1 / 2}-0 g_{9 / 2}$ model space with the interactions (b) slgt0 [57], (c) jun45 [58], and (d) jj44b [59], and the $0 f_{5 / 2}-1 p-0 g_{9 / 2}$ model space with the interactions (e) jun45 [58] and (f) jj44b [59].

not give a consistent picture of the order of the states and, additionally, significantly overestimate excitation energies. It is interesting to note, however, that $\mathrm{jj} 44 \mathrm{~b}$ in the smaller model space [Fig. 6(d)] would agree well with the level ordering of option (i), i.e., $6^{+}$ground state and $2^{+}$isomer. This would also be compatible with the production argument that higher spin states are favored in heavy-ion fusion-evaporation reactions.

Shell-model calculations were repeated using a larger model space, $0 f_{5 / 2}-1 p-0 g_{9 / 2}$, with jun 45 and jj44b interactions; see Fig. 6. The larger model space changed the order of the states as well as produced energy levels closer to experimental results. However, spins of the states are still not in agreement with expectations based on experimental results. Discrepancies between the experimental and theoretical level schemes, as well as between the level schemes obtained with different theoretical model spaces, show that theoretical approaches for this particular nuclide are highly sensitive and cannot offer additional support for the spin assignments.

\section{Mass surface in the region}

The effect of this work on the mass surface was studied via two-neutron $\left(S_{2 n}\right)$ and two-proton $\left(S_{2 p}\right)$ separation energies, and neutron pairing-gap energies $D_{n}$. Two-neutron and twoproton separation energies are sensitive to trends in nuclear structure irrespective of odd-even staggering, making them a useful tool in searching for changes in nuclear deformation and onset of shell closures. Neutron pairing gap energies $D_{n}$ were used as a complementary tool to $S_{2 n}$ and $S_{2 p}$ energies since it is a quantity highly sensitive to pairing of single neutrons.

Two-neutron separation energies in the region of this work are presented in Fig. 7. The new data introduces minor changes to $\mathrm{Zr}, \mathrm{Nb}, \mathrm{Mo}$, and $\mathrm{Tc}$ isotopic chains. The new values offer improved precision compared to [39] and follow the trend set by the literature values. This work extends the $\mathrm{Ru}$ isotopic chain by one isotope, revealing an overestimation of $S_{2 n}$ energy by extrapolated theoretical values.

Two-proton separation energies $S_{2 p}$ (see Fig. 8) largely mirror the changes seen in two-neutron separation energies. As with the $S_{2 n}$ values, minor changes are introduced to $\mathrm{Zr}$, $\mathrm{Nb}, \mathrm{Mo}$, and Tc isotopic chains by the new results. Also, the $\mathrm{Ru}$ chain is again extended by one additional isotope, revealing an underestimation of $S_{2 p}$ energy by extrapolated theoretical values.

The new $S_{2 n}$ and $S_{2 p}$ results suggest minor changes compared to theoretical values in the case of the Ru chain but do not reveal any major changes in the case of previously experimentally known masses.

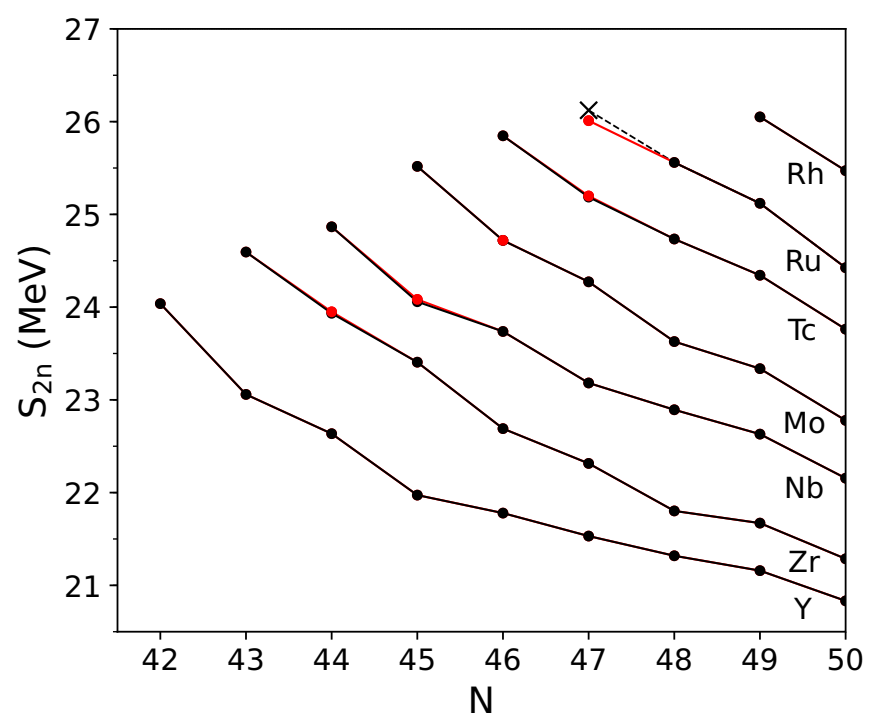

FIG. 7. Two-neutron separation energies $S_{2 n}$. Experimental AME16 [39] values are presented with black dots and results affected by this work with red dots. An extrapolated value for ${ }^{91} \mathrm{Ru}$ adopted from Ref. [39] is presented with an X. 


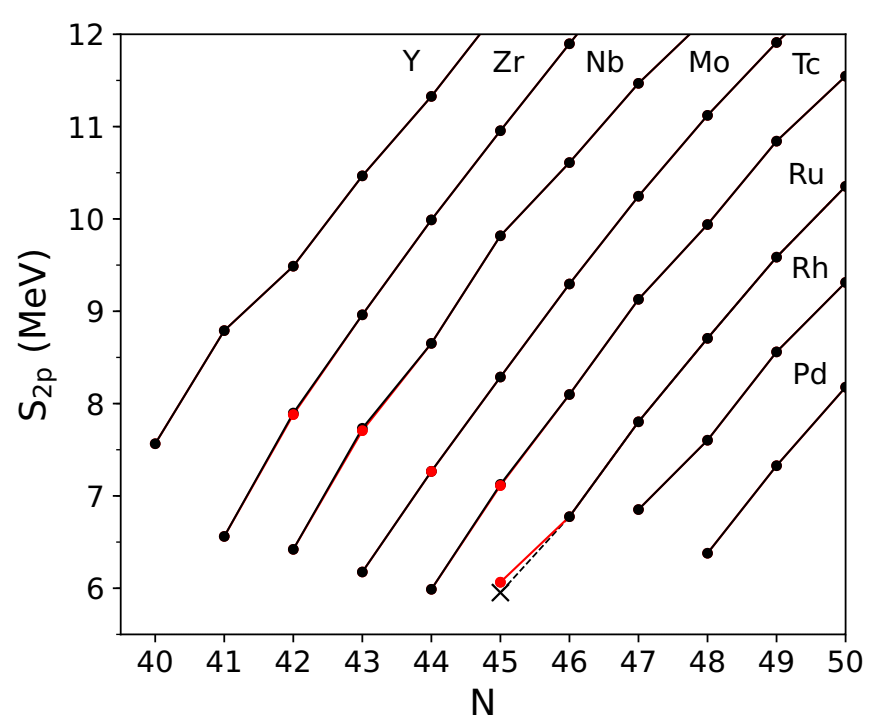

FIG. 8. Two-proton separation energies $S_{2 p}$. Experimental AME16 [39] values are presented with black dots and results affected by this work with red dots. An extrapolated value for ${ }^{89} \mathrm{Ru}$ adopted from Ref. [39] is presented with an X.

Neutron pairing-gap energies

$$
D_{n}=(-1)^{N+1}\left[S_{n}(Z, N+1)-S_{n}(Z, N)\right]
$$

[64], where $S_{n}$ are neutron separation energies, were also studied. $D_{n}$ energies, contrary to $S_{2 n}$ and $S_{2 p}$, are an effective indicator of changes in pairing of individual valence neutrons. This is highlighted by the fact that the neutron pairing gap energy can be expressed using the empirical neutron pairing gap $\Delta^{3}(N)=D_{n}(N) / 2$ which is also known as the odd-even staggering parameter [65]. Neutron pairing gap energies affected by this work are presented in Fig. 9.

Similarly to $S_{2 n}$ and $S_{2 p}$ energies, the neutron pairing gap energies of this work produce only minor changes in $\mathrm{Zr}$, $\mathrm{Nb}$, Mo, and Tc isotopic chains. In the case of ${ }^{90} \mathrm{Ru}$, this work shows a clear overestimation of odd-even staggering by extrapolated values of Ref. [39].

\section{Mass predictions using mirror displacement energies}

In this work, we measured the ground-state mass of ${ }^{89} \mathrm{Ru}$ for the first time. Our result, $-58372(21) \mathrm{keV}$, indicated that it is somewhat (by $112(299) \mathrm{keV}$ ) more bound than the extrapolated literature value, $-58260(298) \mathrm{keV}$ [39]. Since we measured many isospin projection $T_{z}=(N-Z) / 2=+1$ nuclei, we decided to investigate what kind of mass predic-

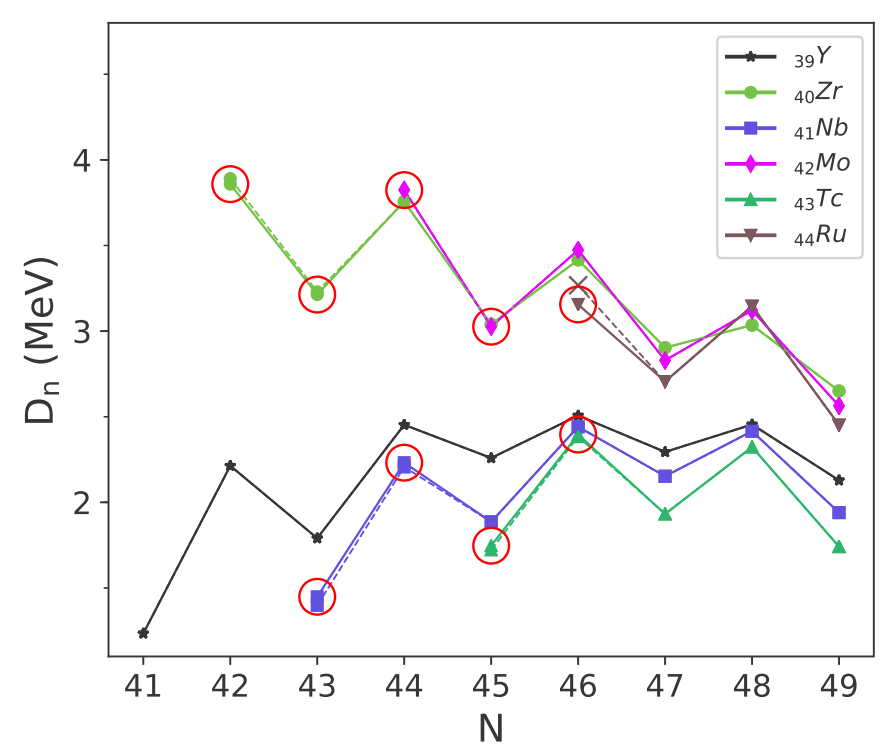

FIG. 9. Neutron pairing-gap energies $D_{n}$. Experimental AME16 [39] values are presented with dashed lines and AME16 values together with results from this work are shown with solid lines. An extrapolated value for ${ }^{90} \mathrm{Ru}$ adopted from Ref. [39] is presented with an X. Results affected by this work are highlighted with red circles.

tions we obtain for the $T_{z}=-1$ mirror partners by combining our precise mass measurements of $T_{z}=+1$ nuclei with the state-of-the-art theoretical calculations for mirror displacement energies (MDE) [3,4]. Such a method was proved to provide accurate predictions for lower mass numbers, such as $A=52$ [3]. The theoretical calculations presented in this work employ extended Skyrme energy density functional $S V_{\mathrm{T} ; \mathrm{NLO}}^{\mathrm{ISB}}$ with proton-neutron-mixed densities and isospin-symmetrybreaking terms in next to leading order [4].

The calculations yield mirror displacement energies of $24290(50)(180) \mathrm{keV}$ for $A=82$ and $25370(50)(180) \mathrm{keV}$ for $A=86$. The uncertainties given in brackets are deduced from the errors of fitted parameters and RMSD, respectively. Predictions for $A=84$ and $A=88$ were not presented since $T_{z}=-1$ partners in these triplets are most likely proton unbound and the model is not suitable for performing calculations of such nuclei. For the same reason, the $T=1 / 2$ doublet at $A=89$ was not studied. The mass excesses of $T_{z}=-1$ isotopes ${ }^{86} \mathrm{Ru}$ and ${ }^{82} \mathrm{Mo}$ were calculated utilizing the results from this work for respective $T_{z}=+1$ mirror nuclei ${ }^{86} \mathrm{Mo}$ and ${ }^{82} \mathrm{Zr}$ (see Table II). The results from this work consistently predict more bound nuclei than literature [39] and also decrease the uncertainty of the predicted mass-excess values.

TABLE II. Mass-excess values $\left(M E_{\mathrm{JYFL}}\right)$ determined in this work, using the measured mass-excess values of ${ }^{82} \mathrm{Zr}$ and ${ }^{86} \mathrm{Mo}$ and the theoretical MDEs, compared with the AME16 [39] values $\left(M E_{\mathrm{AME16}}\right)$, where \# indicates extrapolated values therein. The uncertainties given in brackets are deduced from the errors of fitted parameters and RMSD, respectively. The differences between the two data sets are listed in the last column.

\begin{tabular}{lccccc}
\hline \hline Nuclide & $T_{z}$ & MDE $(\mathrm{keV})$ & $M E_{\mathrm{JYFL}}(\mathrm{keV})$ & $M E_{\mathrm{AME} 16}(\mathrm{keV})$ & $\Delta M E_{\mathrm{JYFL}-\mathrm{AME16}}(\mathrm{keV})$ \\
\hline${ }^{86} \mathrm{Ru}$ & -1 & $25370(50)(180)$ & $-40310(190)$ & $-39770(400) \#$ & $-540(450)$ \\
${ }^{82} \mathrm{Mo}$ & -1 & $24290(50)(180)$ & $-40910(190)$ & $-40370(400) \#$ & $-540(450)$ \\
\hline \hline
\end{tabular}




\section{CONCLUSIONS}

An upgraded system for the production of neutrondeficient isotopes using heavy-ion beams has been successfully commissioned at IGISOL. The new system was used in its first online experiment where a total of six masses were measured: ${ }^{82} \mathrm{Zr},{ }^{84} \mathrm{Nb},{ }^{86} \mathrm{Mo},{ }^{88} \mathrm{Tc},{ }^{88} \mathrm{Tc}^{m}$, and ${ }^{89} \mathrm{Ru}$. The precisions of ${ }^{82} \mathrm{Zr},{ }^{84} \mathrm{Nb}$, and ${ }^{88} \mathrm{Tc}$ were improved and the literature value of ${ }^{86} \mathrm{Mo}$ was verified. ${ }^{88} \mathrm{Tc}^{m}$ and ${ }^{89} \mathrm{Ru}$ were measured for the first time.

The new results for ${ }^{82} \mathrm{Zr}$ and ${ }^{84} \mathrm{Nb}$ do not fully agree with the literature values based on the measurements at the CSRe storage ring in Lanzhou [41]. A thorough comparison between the results from the CSRe and several Penning traps was performed, and a deviation larger than what can be explained by statistical fluctuations alone was discovered. The comparison suggests that Penning trap measurement seem to produce on average about $20 \mathrm{keV}$ higher mass values. The impact on the astrophysical $r p$ process was already studied in Ref. [41]. A systematic shift of around $10 \mathrm{keV}$ will not have a huge impact on the calculated abundances and light curves as it will be partly canceled out in the proton separation energies used as an input for the $r p$-process calculations.

The excitation energy for the long-living isomer in ${ }^{88} \mathrm{Tc}$, $70.4(31) \mathrm{keV}$, was determined for the first time in this work. The order of the lowest three levels in ${ }^{88} \mathrm{Tc}$ was studied based on the excitation energy together with known and expected half-lives for the states. Based on the new limitations, $2^{+}$ is most likely the ground state and $6^{+}$the first isomer in ${ }^{88} \mathrm{Tc}$. This is somewhat unexpected since the ground state was dominantly produced, contrary to the expectation of better production for the higher spin state.

Masses of ${ }^{82} \mathrm{Zr}$ and ${ }^{86} \mathrm{Mo}$ measured in this campaign were used to study corresponding mirror nuclei ${ }^{82} \mathrm{Mo}$ and ${ }^{86} \mathrm{Ru}$ via theoretical MDE's. The resulting mass-excess values are more precise than predicted in the most recent atomic mass evalu- ation for ${ }^{82} \mathrm{Mo}$ and ${ }^{86} \mathrm{Ru}$ [39]. The new values suggest that the studied nuclei are more tightly bound than expected from extrapolations of the mass surface [39]. This is consistent with the observation that the mass of ${ }^{89} \mathrm{Ru}$ measured in this work for the first time was lower than the AME16 extrapolation.

In conclusion, we have improved the knowledge of the mass surface in the neutron-deficient mass region $A=82-89$ by precise Penning-trap measurements. The results indicate that the extrapolated masses might be somewhat overpredicted in this region. Future precision measurements aiming toward $N=Z$ nuclei or beyond are anticipated to shed more light on the evolution of the mass surface in this region rich in isomeric states and structural changes. The recently commissioned PIICR technique will be an invaluable tool for revealing and identifying longer living isomeric states as demonstrated with ${ }^{88} \mathrm{Tc}$ in this work.

\section{ACKNOWLEDGMENTS}

This work has been supported by the Academy of Finland under the Finnish Centre of Excellence Programme 20122017 (Nuclear and Accelerator Based Physics Research at JYFL). A.K., D.N., L.C., and T.E. acknowledge support from the Academy of Finland under Projects No. 275389, No. 284516, No. 312544, No. 295207, and No. 306980. This project has also received funding from the European Union's Horizon 2020 research and innovation program under Grant Agreement No. 771036 (ERC CoG MAIDEN). This work was supported in part by the Polish National Science Centre under Contracts No. 2015/17/N/ST2/04025, No. 2017/24/T/ST2/00159, and No. 2018/31/B/ST2/02220. We acknowledge the CIŚ Świerk Computing Center, Poland, for the allocation of computational resources. J.D. acknowledges support by the STFC Grants No. ST/M006433/1 and No. ST/P003885/1.
[1] B. Cederwall, F. G. Moradi, T. Bäck, A. Johnson, J. Blomqvist, E. Clément, G. de France, R. Wadsworth, K. Andgren, K. Lagergren et al., Nature (London) 469, 68 (2011).

[2] S. Frauendorf and A. Macchiavelli, Prog. Part. Nucl. Phys. 78, 24 (2014).

[3] P. Bączyk, J. Dobaczewski, M. Konieczka, W. Satuła, T. Nakatsukasa, and K. Sato, Phys. Lett. B 778, 178 (2018).

[4] P. Bączyk, W. Satuła, J. Dobaczewski, and M. Konieczka, J. Phys. G: Nucl. Part. Phys. 46, 03LT01 (2019).

[5] C. J. Lister, M. Campbell, A. A. Chishti, W. Gelletly, L. Goettig, R. Moscrop, B. J. Varley, A. N. James, T. Morrison, H. G. Price et al., Phys. Rev. Lett. 59, 1270 (1987).

[6] S. J. Zheng, F. R. Xu, S. F. Shen, H. L. Liu, R. Wyss, and Y. P. Yan, Phys. Rev. C 90, 064309 (2014).

[7] J. Dudek, A. Goźdź, N. Schunck, and M. Miśkiewicz, Phys. Rev. Lett. 88, 252502 (2002).

[8] D. Lunney, J. M. Pearson, and C. Thibault, Rev. Mod. Phys. 75 , 1021 (2003).

[9] H. Schatz, Int. J. Mass Spectrom. 251, 293 (2006).

[10] H. Schatz and W.-J. Ong, Astrophys. J. 844, 139 (2017).

[11] A. Kankainen, S. A. Eliseev, T. Eronen, S. P. Fox, U. Hager, J. Hakala, W. Huang, J. Huikari, D. Jenkins, A.
Jokinen et al. (IS403 Collaboration), Eur. Phys. J. A 25, 129 (2005).

[12] C. Weber, V.-V. Elomaa, R. Ferrer, C. Fröhlich, D. Ackermann, J. Äystö, G. Audi, L. Batist, K. Blaum, M. Block et al., Phys. Rev. C 78, 054310 (2008).

[13] V.-V. Elomaa, G. K. Vorobjev, A. Kankainen, L. Batist, S. Eliseev, T. Eronen, J. Hakala, A. Jokinen, I. D. Moore, Y. N. Novikov et al., Phys. Rev. Lett. 102, 252501 (2009).

[14] E. Haettner, D. Ackermann, G. Audi, K. Blaum, M. Block, S. Eliseev, T. Fleckenstein, F. Herfurth, F. P. Heßberger, S. Hofmann et al., Phys. Rev. Lett. 106, 122501 (2011).

[15] S. Eliseev, K. Blaum, M. Block, A. Dörr, C. Droese, T. Eronen, M. Goncharov, M. Höcker, J. Ketter, E. M. Ramirez, D. A. Nesterenko, Y. N. Novikov, and L. Schweikhard, Appl. Phys. B 114, 107 (2014).

[16] D. A. Nesterenko, T. Eronen, A. Kankainen, L. Canete, A. Jokinen, I. D. Moore, H. Penttilä, S. Rinta-Antila, A. de Roubin, and M. Vilen, Eur. Phys. J. A 54, 154 (2018).

[17] J. Ärje, J. Äystö, H. Hyvönen, P. Taskinen, V. Koponen, J. Honkanen, A. Hautojärvi, and K. Vierinen, Phys. Rev. Lett. 54, 99 (1985).

[18] J. Äystö, Nucl. Phys. A 693, 477 (2001). 
[19] I. D. Moore, P. Dendooven, and J. Ärje, Hyperfine Int. 223, 17 (2013).

[20] J. Huikari, P. Dendooven, A. Jokinen, A. Nieminen, H. Penttilä, K. Peräjärvi, A. Popov, S. Rinta-Antila, and J. Äystö, Nucl. Instrum. Meth. Phys. Res. B 222, 632 (2004).

[21] V.-V. Elomaa, Ph.D. thesis, University of Jyväskylä, Jyväskylä, Finland, 2009.

[22] R. Béraud, A. Emsallem, A. Astier, R. Bouvier, R. Duffait, Y. L. Coz, S. Morier, A. Wojtasiewicz, Y. Lazarev, I. Shirokovsky et al., Nucl. Instrum. Meth. Phys. Res. A 346, 196 (1994).

[23] P. Karvonen, I. Moore, T. Sonoda, T. Kessler, H. Penttilä, K. Peräjärvi, P. Ronkanen, and J. Äystö, Nucl. Instrum. Meth. Phys. Res. B 266, 4794 (2008).

[24] I. Moore, T. Eronen, D. Gorelov, J. Hakala, A. Jokinen, A. Kankainen, V. Kolhinen, J. Koponen, H. Penttilä, I. Pohjalainen et al., Nucl. Instrum. Meth. Phys. Res. B 317, 208 (2013).

[25] O. B. Tarasov and D. Bazin, Nucl. Instrum. Meth. Phys. Res. B 266, 4657 (2008).

[26] N. Wang and W. Scheid, Phys. Rev. C 78, 014607 (2008).

[27] A. V. Karpov, A. S. Denikin, M. A. Naumenko, A. P. Alekseev, V. A. Rachkov, V. V. Samarin, V. V. Saiko, and V. I. Zagrebaev, Nucl. Instrum. Meth. Phys. Res. A 859, 112 (2017).

[28] A. Nieminen, J. Huikari, A. Jokinen, J. Äystö, P. Campbell, and E. Cochrane, Nucl. Instrum. Meth. Phys. Res. A 469, 244 (2001).

[29] T. Eronen, V. S. Kolhinen, V. V. Elomaa, D. Gorelov, U. Hager, J. Hakala, A. Jokinen, A. Kankainen, P. Karvonen, S. Kopecky et al., Eur. Phys. J. A 48, 46 (2012).

[30] G. Gräff, H. Kalinowsky, and J. Traut, Z. Phys. A 297, 35 (1980).

[31] M. König, G. Bollen, H.-J. Kluge, T. Otto, and J. Szerypo, Int. J. Mass Spectrom. Ion Proc. 142, 95 (1995).

[32] G. Savard, S. Becker, G. Bollen, H.-J. Kluge, R. Moore, T. Otto, L. Schweikhard, H. Stolzenberg, and U. Wiess, Phys. Lett. A 158, 247 (1991).

[33] M. Kretzschmar, Int. J. Mass Spectrom. 264, 122 (2007).

[34] S. George, K. Blaum, F. Herfurth, A. Herlert, M. Kretzschmar, S. Nagy, S. Schwarz, L. Schweikhard, and C. Yazidjian, Int. J. Mass Spectrom. 264, 110 (2007).

[35] A. Kellerbauer, K. Blaum, G. Bollen, F. Herfurth, H.-J. Kluge, M. Kuckein, E. Sauvan, C. Scheidenberger, and L. Schweikhard, Eur. Phys. J. D 22, 53 (2003).

[36] L. Canete, Ph.D. thesis, Jyväskylä, Finland, 2019.

[37] R. T. Birge, Phys. Rev. 40, 207 (1932).

[38] D. Nesterenko, M. Vilén, T. Eronen, A. Kankainen et al. (unpublished).

[39] M. Wang, G. Audi, F. Kondev, W. Huang, S. Naimi, and X. Xu, Chin. Phys. C 41, 030003 (2017).

[40] G. Audi, F. G. Kondev, M. Wang, W. Huang, and S. Naimi, Chin. Phys. C 41, 030001 (2017).

[41] Y. Xing, K. Li, Y. Zhang, X. Zhou, M. Wang, Y. Litvinov, K. Blaum, S. Wanajo, S. Kubono, G. Martínez-Pinedo et al., Phys. Lett. B 781, 358 (2018).

[42] X. Tu, M. Wang, Y. Litvinov, Y. Zhang, H. Xu, Z. Sun, G. Audi, K. Blaum, C. Du, W. Huang et al., Nucl. Instrum. Meth. Phys. Res. A 654, 213 (2011).

[43] X. Xu, P. Zhang, P. Shuai, R. J. Chen, X. L. Yan, Y. H. Zhang, M. Wang, Y. A. Litvinov, H. S. Xu, T. Bao et al., Phys. Rev. Lett. 117, 182503 (2016).
[44] P. Zhang, X. Xu, P. Shuai, R. Chen, X. Yan, Y. Zhang, M. Wang, Y. Litvinov, K. Blaum, H. Xu et al., Phys. Lett. B 767, 20 (2017).

[45] Y. H. Zhang, P. Zhang, X. H. Zhou, M. Wang, Y. A. Litvinov, H. S. Xu, X. Xu, P. Shuai, Y. H. Lam, R. J. Chen et al., Phys. Rev. C 98, 014319 (2018).

[46] C. Yazidjian, G. Audi, D. Beck, K. Blaum, S. George, C. Guénaut, F. Herfurth, A. Herlert, A. Kellerbauer, H.-J. Kluge, D. Lunney, and L. Schweikhard, Phys. Rev. C 76, 024308 (2007).

[47] S. George, G. Audi, B. Blank, K. Blaum, M. Breitenfeldt, U. Hager, F. Herfurth, A. Herlert, A. Kellerbauer, H.-J. Kluge et al., Europhys. Lett. 82, 50005 (2008).

[48] T. Kurtukian Nieto, J. Souin, T. Eronen, L. Audirac, J. Äystö, B. Blank, V. V. Elomaa, J. Giovinazzo, U. Hager, J. Hakala et al., Phys. Rev. C 80, 035502 (2009).

[49] A. Kankainen, T. Eronen, D. Gorelov, J. Hakala, A. Jokinen, V. S. Kolhinen, M. Reponen, J. Rissanen, A. Saastamoinen, V. Sonnenschein, and J. Äystö, Phys. Rev. C 89, 051302(R) (2014).

[50] D. A. Nesterenko, A. Kankainen, L. Canete, M. Block, D. Cox, T. Eronen, C. Fahlander, U. Forsberg, J. Gerl, P. Golubev et al., J. Phys. G: Nucl. Part. Phys. 44, 065103 (2017).

[51] A. Kankainen, V.-V. Elomaa, T. Eronen, D. Gorelov, J. Hakala, A. Jokinen, T. Kessler, V. S. Kolhinen, I. D. Moore, S. Rahaman et al., Phys. Rev. C 82, 034311 (2010).

[52] A. A. Valverde, M. Brodeur, G. Bollen, M. Eibach, K. Gulyuz, A. Hamaker, C. Izzo, W.-J. Ong, D. Puentes, M. Redshaw et al., Phys. Rev. Lett. 120, 032701 (2018).

[53] J. Fallis, J. A. Clark, K. S. Sharma, G. Savard, F. Buchinger, S. Caldwell, A. Chaudhuri, J. E. Crawford, C. M. Deibel, S. Gulick et al., Phys. Rev. C 84, 045807 (2011).

[54] C. Guénaut, G. Audi, D. Beck, K. Blaum, G. Bollen, P. Delahaye, F. Herfurth, A. Kellerbauer, H.-J. Kluge, J. Libert et al., Phys. Rev. C 75, 044303 (2007).

[55] P. Schury, C. Bachelet, M. Block, G. Bollen, D. A. Davies, M. Facina, C. M. Folden III, C. Guénaut, J. Huikari, E. Kwan et al., Phys. Rev. C 75, 055801 (2007).

[56] J. Savory, P. Schury, C. Bachelet, M. Block, G. Bollen, M. Facina, C. M. Folden, C. Guénaut, E. Kwan, A. A. Kwiatkowski et al., Phys. Rev. Lett. 102, 132501 (2009).

[57] H. Herndl and B. Brown, Nucl. Phys. A 627, 35 (1997).

[58] M. Honma, T. Otsuka, T. Mizusaki, and M. Hjorth-Jensen, Phys. Rev. C 80, 064323 (2009).

[59] B. A. Brown and A. F. Lisetskiy (unpublished); see also endnote 28 in B. Cheal, E. Mané, J. Billowes, M. L. Bissell, K. Blaum, B. A. Brown, F. C. Charlwood, K. T. Flanagan, D. H. Forest, C. Geppert et al., Phys. Rev. Lett. 104, 252502 (2010).

[60] D. Rudolph, F. Cristancho, C. J. Gross, A. Jungclaus, K. P. Lieb, M. A. Bentley, W. Gelletly, J. Simpson, H. Grawe, J. Heese et al., J. Phys. G: Nucl. Part. Phys. 17, L113 (1991).

[61] A. Odahara, Y. Gono, S. Mitarai, T. Shizuma, E. Ideguchi, J. Mukai, H. Tomura, B. J. Min, S. Suematsu, T. Kuroyanagi et al., Z. Phys. A 354, 231 (1996).

[62] A. B. Garnsworthy, P. H. Regan, S. Pietri, Y. Sun, F. R. Xu, D. Rudolph, M. Górska, L. Cáceres, Zs. Podolyák, S. J. Steer et al., Phys. Rev. C 80, 064303 (2009).

[63] R. Gross and A. Frenkel, Nucl. Phys. A 267, 85 (1976).

[64] B. A. Brown, Phys. Rev. Lett. 111, 162502 (2013).

[65] W. Satuła, J. Dobaczewski, and W. Nazarewicz, Phys. Rev. Lett. 81, 3599 (1998). 